\title{
INDUCED COUPLING: AN APPROACH TO MODELING AND MANAGING COMPLEX HUMAN-LANDSCAPE INTERACTIONS
}

\author{
Nicholas R. Magliocea \\ Master's of Environmental Management Candidate, Nicholas School of the \\ Environment and Earth Sciences, Duke University, Durham, North Carolina \\ nrm@duke.edu
}

\begin{abstract}
Population growth and overdevelopment are driving complicated interactions between human and natural systems. A preliminary investigation of human and natural systems as hierarchical, complex systems suggests that problematic, emergent behaviors are the result of 'induced coupling' between the two systems. Human manipulations aimed at mitigating natural disasters often change how human and natural systems interact in space and time. The dynamics of each separate system are fundamentally altered when they become coupled. This coupling drives unexpected, emergent behaviors through the introduction of strong, non-linear feedbacks.

Human-landscape interactions present a unique challenge because of the mismatch in scales between coupled processes. Induced coupling describes problematic system behaviors (i.e. natural disasters intensified by human actions) as the product of coupling short-term human activities to long-term natural processes. As a descriptive model, induced coupling draws upon the conceptualization of human and natural systems as hierarchical, complex systems, and it utilizes the systems dynamics approach to identify delays introduced by human manipulations. Induced coupling aides in the description and analysis of coupled human-landscape interactions, and provides a focus for numerical modeling and identification of leverage points for management actions.
\end{abstract}

Keywords: Induced coupling, human-landscape interactions, hierarchical systems

\section{INTRODUCTION}

A new class of environmental problem has emerged that is far more complex than traditional ones. Population growth and overdevelopment are driving complex interactions between human systems and natural landscapes (e.g. Hooke, 1994; 2000; Haff, 2003). Humans have been altering the natural landscape for millennia (e.g. Pyne, 2001; Fagan, 2004), but the scale of human manipulations today is larger than ever before (Vitousek et. al., 1997). Population growth within urban centers has prompted expansive urban sprawl around cities throughout the world (Davis, 2006). As a result, humans are in much closer proximity to natural hazards than ever before. For example, the number of acres burned by wildfires in the Los Angeles Basin has not changed significantly over the last 40 years, yet both dollar damage and number of structures lost have increased exponentially (Magliocca, 2006). Humans have responded by manipulating the natural landscape with extensive protective measures in an effort to mitigate natural disasters. The resulting human-landscape interactions 
become overwhelmingly complex, masking the role human activities play in intensifying damage from natural hazards.

Traditional environmental problems involve linear, one-way interactions between humans and natural landscapes. Human impacts on natural processes are typically characterized by short-lived changes to the local environment, without lasting changes to the way humans interact with the landscape (Werner and McNamara, in press). Negative impacts emerge on the same temporal and spatial scale from which they were created. In other words, the negative effects of human impacts on natural systems are localized in time and space-- like pollution in a river. Pollution enters a river and organisms that come into contact with that pollution become sick. If more pollutant enters the river, then correspondingly more organisms become ill. A clear relationship exists in which a given amount of pollutant can affect a given amount of people within a localized area. Furthermore, the processes through which negative effects emerge operate on the same time scales as the processes through which humans created those effects. For example, a given amount of pollutant is released into a river per second, and that pollutant chemically reacts with an organism's biology at a rate on the order of seconds. Granted, a time delay may exist between the initial human impact (release of pollutant) and its reaction with a natural system (finding its way into an organism), but the linear cause-and-effect pathway still operates on the same time scale. Even large-scale problems, such as the ozone layer hole, fall into the category on one-way human interactions with natural systems. Although on a global scale, the introduction of a given amount of CFC's into the atmosphere per second corresponds to a proportional amount of ozone destroyed per second. The processes of CFC introduction and ozone destruction operate on the same time scale, and are clearly linked through a linear cause-and-effect chain. Because induced coupling links processes on different time scales, the cause-and-effect chain is never linear. Rather, negative impacts from human activities manifest themselves on the longer time scale of natural processes.

Another reason traditional environmental problems are classified as linear, one-way phenomenon is that their problematic behaviors (or dynamics) are generated external to the natural system that was impacted. Negative effects from human impacts are driven by external inputs into the natural system which become disruptive as that system becomes impaired. For instance, the ozone hole is a problem because of increased levels of radiation exposure on the planet's surface. However, ultraviolet radiation is an external, one-way input into the global system, and is itself unchanged by human impacts to the ozone layer. The internal dynamics of the natural system, in this case the ozone layer, have been altered, but in such a way that the negative impacts are the product of external inputs. The chemical reactions between CFCs and ozone molecules are not harmful, but the increase of ultraviolet radiation that results is detrimental. The same line of reasoning applies to river pollution as well. It is the pollutant that is harming organisms and not altered river processes such as flow routing. This is not the case for many emerging environmental problems, which are generated by the internal dynamics of coupled human and natural systems rather than exclusively external forces.

\section{INDUCED COUPLING}

Today's new class of environmental problems are distinct because their problematic behaviors are generated internally through a phenomenon referred to as induced 
coupling. Induced coupling is a phenomenon in which short-term, small-scale human activities become linked to and influence long-term, large-scale behaviors of natural systems. This is done through human manipulations to natural landscapes that create new and alter existing feedbacks within the natural system in such a way that changes the dynamics of that system. These interactions are distinct from traditional humanlandscape interactions because they are nonlinear. In other words, the actions of one component or system influence and are influenced by the behavior of another. Thus, at the heart of induced coupling, is the fact that human activities can influence and are influenced by the behavior of natural systems through the initiation of coupled, nonlinear interactions. In general, natural disasters, such as floods or wildfires, can be intensified when human and natural systems become coupled in such a way that small, short-term human manipulations initiate two-way interactions with longer-term natural processes.

Again, take the case of wildfires in the Los Angeles Basin as an example. The natural fire regime in Southern California is characterized by small, frequent fires that burn fire-adapted vegetation called chaparral. These frequent fires are a key ecological process that serve to thin-out dead plant mass, clear areas for young chaparral plants to thrive, and fertilize the soil. During certain times of the year, larger fires can occur driven by strong, dry Santa Ana winds. These fires typically burn large areas that regenerate slowly, which act as natural fires breaks to future fires by creating a swath of fairly inflammable vegetation. The mountains surrounding Los Angeles are of this type of fire-dependent ecosystem, but unfortunately are also highly-valued housing sites for their views and proximity to nature. As development into the natural landscape advances, property will inevitably be damaged by wildfires. The human response is to suppress any future fires that may threaten property. With property safe from frequent fires, opportunities are opened for further development, which in turn needs increased fire suppression efforts to protect the increased human presence. Through human manipulation of the natural landscape, a short-term, small-scale iterative feedback loop has been created that promotes development by excluding small, frequent fires (seen as dashed lines in Figure 1). This is induced coupling. Fires act as a process of dissipation in this natural system, but that process is disrupted by human manipultations.

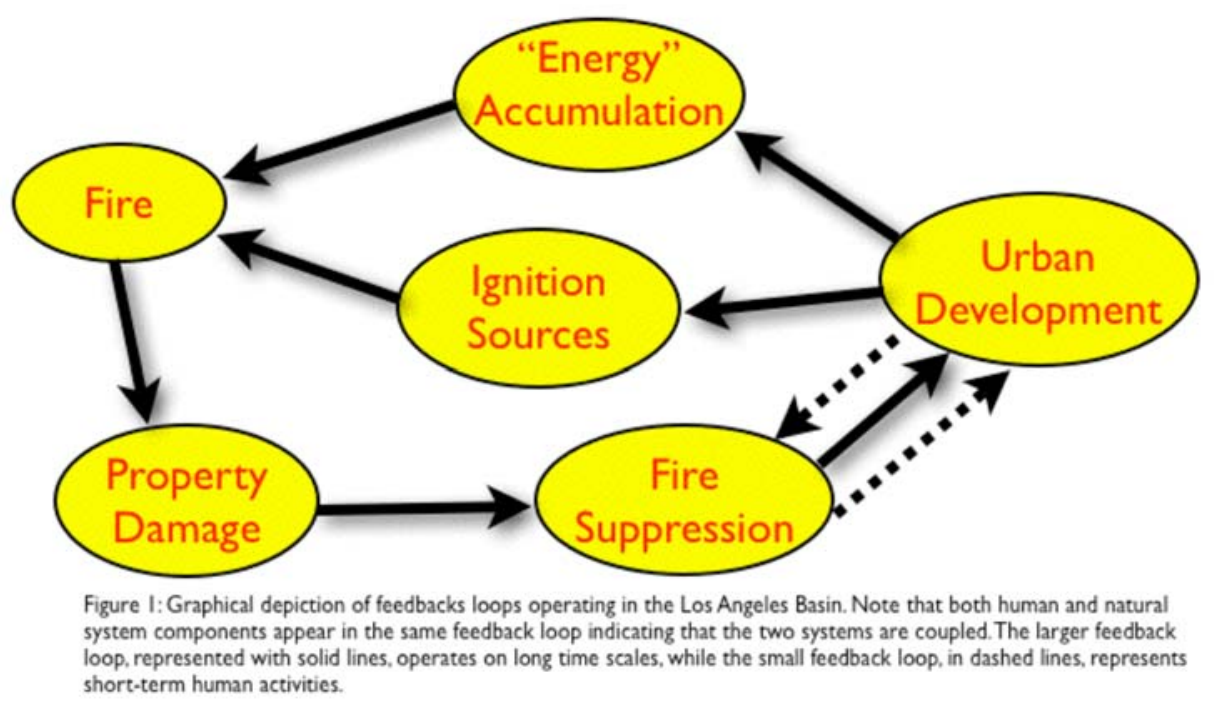


As a consequence, the accumulation of fuels combined with more ignition sources from a greater human presence can lead to catastrophic property damage from intensified fires. Property damage is worse than it would have otherwise been naturally for two reasons: 1) the exclusion of regular, dissipative fires allowed for greater development of areas close to a natural wildfire hazard, and 2) wildfires were intensified by the unnatural build-up of fuels. Destructive natural system behavior was generated internally by altering the longer-term dynamics of a fire-adapted ecosystem through short-term human manipulations.

Induced coupling entails fundamentally changing the way in which human and natural systems interact in space and time. This can be done by physically establishing new spatial boundaries for natural processes. In the case of Southern California fire suppression, the encroachment of development into 'wildlands' limits the spatial extent that wildfires are allowed to burn. Fires are limited to the steepest slopes, which are unsuitable for building but exhibit the areas of fastest fire spread. In addition, the natural fire frequency is re-scaled by excluding smaller, frequent fires and promoting less frequent, albeit larger, fire events. The fundamental behavior of a natural process, fire in this case, has been altered by human manipulations. Fires are spreading faster, burning more intensely, and spreading over larger areas as a result. By re-scaling natural patterns and processes in time and space, human manipulations can alter the dynamics of natural systems, which produce new internal feedbacks that drive problematic behaviors.

Protective measures instituted to mitigate natural disasters often have the unexpected consequence of linking short-term human activities to longer-term natural processes. The effect of coupling systems results in more intense impacts to human systems than would have otherwise occurred naturally. When natural forces finally overcome protective measures, the damage is much worse than it otherwise would have been without protective measures that disrupted natural processes to allow development. For example, extensive levee systems protect against high-frequency, small-scale flooding events and allow for development. However, a large flood that over-tops levees transports larger quantities of water much more quickly than if floodwaters had been able to dissipate upstream over the flood plain. Property damage is much more extensive not only because the flood was intensified by channelization of the river, but also because of the increased human presence in a naturally hazardous area. Induced coupling creates complex environmental problems because it initiates and drives an iterative cycle that magnifies an initial disruption of natural processes. A filtering-out of small, frequent events on short time scales occurs, which drives the emergence of intensified natural processes on longer time scales.

\section{DYNAMICS OF HIERARCHICAL COMPLEX SYSTEMS}

An investigation within the frameworks of hierarchical complex systems and systems dynamics reveals how induced coupling between human and natural systems drives pervasive, destructive behaviors. Both human and natural systems can be described using a hierarchical complex systems model that includes sets of descriptive variables for each level of spatial and temporal organization (Werner and McNamara, in press). 


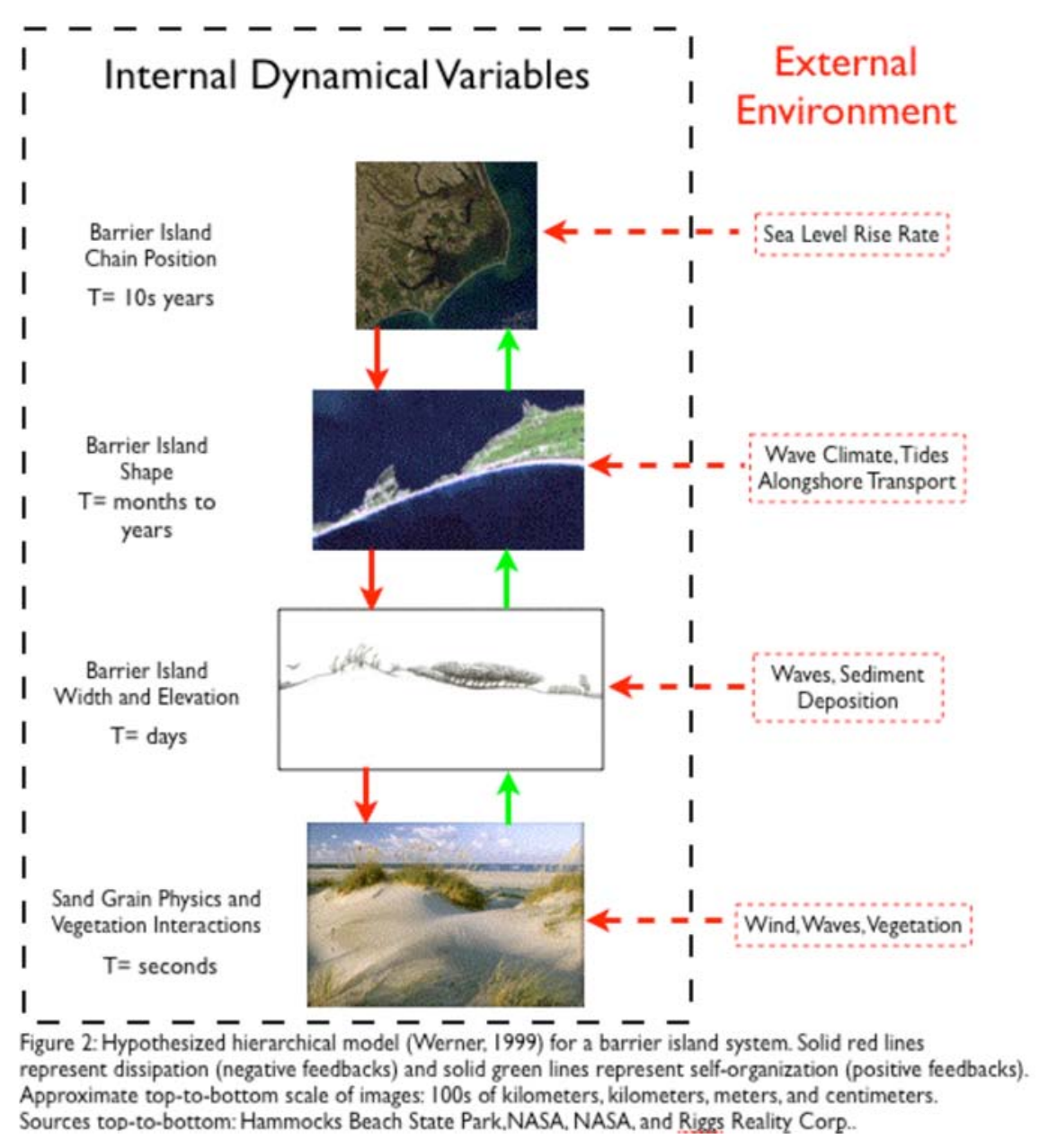

System parameters or components can be organized into discrete levels within a hierarchy according to their response time (time scale) to perturbation (Werner, 2003). For example, individual sand grains interact to build dunes, but their motions are ultimately slaved to the interactions between winds and the larger-scale shape of the dune that drive either accumulation or erosion. (Figure 2). In the case of human systems, the real estate market determines the day-to-day price of a house primarily on local factors, but also responds to longer-term trends of regional housing demand. A hierarchical complex systems analysis implies that a description of a system's dynamics that operate on large spatial scales necessarily entails long time scales, and the same is true for frequent, small-scale dynamics.

Natural landscapes provide clear illustrations of how overall system behaviors can be understood as interactions between internal dynamic variables and external environmental inputs (Figure 2). In the case of coupled human-landscape interactions, human manipulations often provide inputs that alter the internal dynamics of natural processes. Patterns in natural landscapes are the product of the dynamic interplay of positive and negative feedbacks between a hierarchy spatial and temporal scales (Werner, 1999). Variables and processes at large spatial and slow temporal scales, at the top of the hierarchical model, provide a context for interactions at smaller spatial and slower time scales. Positive feedbacks between components of the same spatial and temporal scale promote self-organization of those components into higher levels of emergent order. Negative feedbacks act to dissipate that order by slaving individual 
components to processes at larger spatial and longer time scales. Dynamic, nonlinear interactions between processes of self-organization and dissipation drive the overall behavior of complex systems.

Human manipulations of natural landscapes, however, introduce delays into the dissipation of short-term, small-scale dynamics and push the system away from steady state. In this context, the steady state of an ecosystem is considered to be a description of the persistent states of variables that constitute an ecosystem's structures and processes. Manipulations of natural processes perturb the natural system away from an established steady state through the delay of negative feedbacks/dissipative processes and the promotion of altered positive feedbacks. This destabilizes the system's state prompting either a return to its original steady state or a transition to a new steady state with impetus from unchecked positive feedbacks. Returning to the wildfire example, the established steady state consists of well-adapted communities of organisms that thrive in an ecosystem subjected to regular fire events. Positive feedbacks come in the form of maturation of chaparral vegetation which provides the fuel for ecosystem-maintaining fires. The more fuel that is available, the more likely it is that a fire will ignite and spread. Negative feedbacks are the fires themselves that dissipate bulky, dead chaparral vegetation and recycle accumulated nutrients back into the soil. However, fire suppression efforts disrupt the negative/dissipative feedbacks of regular fire events for the purpose of protecting development. Fire suppression re-scales the frequency of fire events and pushes the fire-adapted ecosystem further away from normal ecosystem function. As a result, positive feedbacks go unchecked and heavy fuel loads accumulate. Thus, the only way the natural system can return to its previous steady state is through very large and intense fires that typically consume landscape-scale areas of chaparral vegetation. Not only do fire events occur less frequently, but they have also become more severe. Human manipulations have disrupted negative feedbacks allowing unnatural accumulations of fuel. When human activities become intertwined with natural processes, the very nature of those processes can be altered. In this manner, human manipulations have the power to influence large-scale, longer-term natural system behaviors by extensively disrupting feedbacks from short-term, localized processes.

\section{DISCUSSION}

Solutions to this new class of environmental problems are often unknown or prohibitively difficult/expensive to implement. This is due to the nonlinear nature of ecosystem processes and the delays that human manipulations introduce into them. Natural processes that adversely affect human systems on large scales often operate on long time scales. Therefore, when human manipulations become intertwined with natural processes, environmental problems do not become apparent until well after the initiation of disruptive activities. This suggests that human activities and natural landscape processes can no longer be treated as separate systems, but rather as potentially coupled, interconnected systems.

The concept of induced coupling provides a framework from which to describe and analyze human-landscape interactions. Induced coupling allows for framing the problem of destructive human-landscape interactions in terms of human manipulations within a hierarchy of natural processes. This has many implications for both modeling and managing coupled human-landscape interactions. Previous approaches to modeling human and natural systems typically treat them as separate 
and contained within a time and spatial scale of interest. From the perspective of induced coupling, the focus of modeling becomes the interdependencies created by coupling the two systems and the long-term behaviors that result. Modeling in this manner allows for the exploration of interactions between the two systems in a dynamic setting. The model can be manipulated to investigate the effects of various management strategies on time scales that would not be feasible for conventional lab or field research. This approach enables an articulation of the dynamics of humanlandscape interactions on multiple spatial and temporal scales, which can elucidate the forces driving problematic interactions.

For managers, the goal of this mode of inquiry is to facilitate an understanding that the relationship between human and natural systems goes beyond short-term management efforts. Specifically, managers must realize that the natural system dynamics that produce landscape-scale behaviors can be altered by coupling them to management activities. The current approach to environmental problems typically divides coupled systems into individual system components to create a management plan for the whole. While this may be easier logistically, it fails to capture the synergistic relationships between each of the components and their environment on multiple temporal and spatial scales. A failure to recognize coupled interactions exists because they are often hidden by the more immediate behaviors of the system's components. Individual components, or a "package" of determinants, that seem unconnected can form a synergistic convergence and become coupled once they interact (Corning, 2003). The proposed concept of induced coupling would require shifting the management paradigm to one that recognizes coupled interactions as organizational drivers and leverage points for action. The hope is that this approach engages managers in a policy design process that aims to reconstruct or uncouple destructive human-landscape interactions.

\section{REFERENCES}

Corning, P. (2003). Nature's Magic: Synergy In Evolution and the Fate of Humankind. Cambridge UP, Cambridge, UK.

Davis, M. (2006). Planet of Slums, Verso, London, 228p.

Fagan, B. (2004). The Long Summer: How Climate Changed Civilization. Basic Books, New York.

Haff, J.K. (2003). "Neogeomorphology, Prediction and the Anthropic Landscape". In P.R. Wilcock and R.M. Iverson (eds.), Prediciton in Geomorphology, Geophysical Monograph, 135, AGU Press, 15-26.

Hooke, R.LeB. (1994). "On the Efficacy of Humans As Geomorphic Agents." GSA Today, 4, 217-225.

Magliocca, N.R. (2006). "Managing Overwhelming Complexity in Human-landscape Interactions". Proceedings of the 50th Annual International Society for the Systems Science Conference, July 9-14, 2006. Sonoma State University.

Pyne, S. (2001). A Brief History of Fire. University of Washington Press, Seattle.

Vitousek, P.M., Mooney, H.A., Lubchenco, J. and Melillo, J.M. (1997). "Human domination of Earth's ecosystems." Science, 277, 494-499.

Werner, B.T. (1999). "Complexity in Natural Landform Patterns." Science, 284, 102105.

Werner, B.T. (2003). "Modeling landforms as self-organized, hierarchical dynamical systems", in Prediction in Geomorphology, eds P.R. Wilcock and R.M. Iverson, 
Induced Coupling

Geophysical Monograph 135, American Geophysical Union Press, Washington, D.C., 133-150.

Werner, B.T. and McNamara, D.E. (in press). "Dynamics of Coupled HumanLandscape Systems”. PNAS. Contact B.T. Werner (bwerner@ucsd.edu).

\section{Figures}

Hammocks Beach State Park.

http://ils.unc.edu/parkproject/visit/habe/hmxbeach/Artwork/New\%20Folder/profileha be.png

NASA. http://www.camex4.com/photo0160.html

NASA. http://www.answers.com/topic/outer-banks

Riggs Reality Corporation. http://riggsrealtycorp.com/assests/homeimage1.jpg. 